\title{
Naegleria fowleri Induces Jurkat T Cell Death via O-deGIcNAcylation
}

\author{
Young Ah Lee $\mathbb{0}$, Kyeong Ah Kim, Myeong Heon Shin* $\mathbb{C}^{\circ}$ \\ Department of Environmental Medical Biology, Institute of Tropical Medicine, Yonsei University College of Medicine, Seoul 03722, Korea
}

\begin{abstract}
The pathogenic free-living amoeba Naegleria fowleri causes primary amoebic meningoencephalitis, a fatal infection, by penetrating the nasal mucosa and migrating to the brain via the olfactory nerves. $N$. fowleri can induce host cell death via lytic necrosis. Similar to phosphorylation, O-linked $\beta-\mathrm{N}$-acetylglucosamine (O-GlcNAc) glycosylation (OGlcNAcylation) is involved in various cell-signaling processes, including apoptosis and proliferation, with O-GlcNAc addition and removal regulated by O-GlcNAc transferase and O-GlcNAcase (OGA), respectively. However, the detailed mechanism of host cell death induced by $N$. fowleri is unknown. In this study, we investigated whether $N$. fowleri can induce the modulation of O-GlcNAcylated proteins during cell death in Jurkat T cells. Co-incubation with live N. fowleri trophozoites increased DNA fragmentation. In addition, incubation with $N$. fowleri induced a dramatic reduction in O-GlcNAcylated protein levels in 30 min. Moreover, pretreatment of Jurkat T cells with the OGA inhibitor PUGNAc prevented N. fowleri-induced O-deGlcNAcylation and DNA fragmentation. These results suggest that O-deGlcNAcylation is an important signaling process that occurs during Jurkat T cell death induced by N. fowleri.
\end{abstract}

Key words: Naegleria fowleri, O-GlcNAcylation, host cell death

Naegleria fowleri is a free-living amoeba found in freshwater, soil, hot springs, and poorly chlorinated swimming pools [1]. In humans, N. fowleri trophozoites cause primary amoebic meningoencephalitis by penetrating the nasal mucosa and migrating to the brain via the olfactory nerves [1,2]. Naegleria can induce acute and severe tissue inflammatory responses associated with lytic necrosis [2], and N. fowleri can cause host cell death in both contact-dependent and contact-independent manners [2,3]. It has also been reported that $N$. fowleri induces reactive oxygen species-dependent necroptosis in host cells [4]. Interestingly, N. fowleri did not induce the cleavage of caspase 3 of Jurkat T cells in co-culture system [4]. Although host cell death is an important aspect of pathogenesis in infection with N. fowleri, the detailed mechanisms involved in N. fowleri-induced host cell death are still not fully understood.

O-linked $\beta$-N-acetylglucosamine (O-GlcNAc) glycosylation (O-GlcNAcylation) is the main form of glycosylation in the cytosolic and nuclear compartments of eukaryotic cells [5]. Similar to phosphorylation, O-GlcNAcylation is a reversible

\footnotetext{
• Received 30 July 2021, revised 16 September 2021, accepted 17 September 2021. *Corresponding author (myeong@yuhs.ac) (C) 2021, Korean Society for Parasitology and Tropical Medicine This is an Open Access article distributed under the terms of the Creative Commons Attribution Non-Commercial License (https://creativecommons.org/licenses/by-nc/4.0) which permits unrestricted non-commercial use, distribution, and reproduction in any medium, provided the original work is properly cited.
}

post-translational modification in which O-GlcNAc is added to serine or threonine residues of nuclear and cytoplasmic proteins [5,6]. O-GlcNAcylation of proteins is also linked to glucose metabolism because O-GlcNAcylation is an end point of the hexosamine biosynthetic pathway $[8,10]$. In mammals, O-GlcNAcylation is regulated by only 2 enzymes, O-GlcNAc transferase (OGT) and O-GlcNAcase (OGA), which catalyze the addition and removal of O-GlcNAc, respectively [5,6]. OGlcNAcylation regulates various cellular processes such as apoptosis, cell proliferation, transcription, translation, and signal transduction [5]. An increase in O-GlcNAcylation is associated with cell proliferation and survival, whereas a decrease (O-deGlcNAcylation) is related to cell cycle arrest and cell death [6-8]. Recently, it was reported that Entamoeba histolytica induces O-deGlcNAcylation of proteins in HepG2 cells during cell death [9]. However, the signaling role of O-GlcNAcylation in host cell death induced by $N$. fowleri is unknown. In this study, we investigated whether the modulation of O-GlcNAcylation is an important signaling event in N. fowleri-induced Jurkat $\mathrm{T}$ cell death.

N. fowleri trophozoites (Carter NF69; ATCC No. 30215) were cultured under axenic conditions in Nelson's medium at $37^{\circ} \mathrm{C}$. Jurkat T cells (clone E6-1; ATCC No. TIB-152TM) were grown in RPMI 1640 medium (Gibco/Invitrogen, Gaithersburg, MD, USA) with $10 \%$ fetal bovine serum (Gibco/Invitrogen) in a 
humidified $5 \% \mathrm{CO}_{2}$ incubator at $37^{\circ} \mathrm{C}$.

To observe $N$. fowleri-induced cell death caused by DNA fragmentation, Jurkat T cells $\left(4 \times 10^{6}\right.$ cells/sample $)$ were incubated with $N$. fowleri trophozoites at a ratio of 1:2 or 1:1 (Jurkat T cells to N. fowleri) for 3 or $6 \mathrm{hr}$ at $37^{\circ} \mathrm{C}$ in a humidified $\mathrm{CO}_{2}$ incubator. After incubation, the cells were harvested by centrifugation and washed with cold phosphate-buffered saline (PBS). DNA was extracted using a TaKaRa kit (MK600; Shiga, Japan) according to the manufacturer's protocol. DNA samples were separated by electrophoresis on a $2 \%$ agarose gel and visualized using ethidium bromide to assess DNA fragmentation. For $\mathrm{LDH}$ release assay, Jurkat T cells $\left(2 \times 10^{5}\right.$ cells/ sample) pretreated with or without PUGNAc were incubated with live $\mathrm{N}$. fowleri for $3 \mathrm{hr}$ at a 1:1 ratio (Jurkat $\mathrm{T}$ cells to $\mathrm{N}$. fowleri). After incubation, LDH release was determined by evaluating the amounts of LDH in culture supernatants using the CytoTox 96 cytotoxicity assay system (Promega, Madison, WI, USA) according to manufacturer protocol. The background (spontaneous LDH release) value was measured in non-stimulated cells and subtracted from each measurement. Maximum LDH release was measured by incubating nonstimulated cells in a lysis solution (1\% Triton X-100 and PBS) at $37^{\circ} \mathrm{C}$ for $45 \mathrm{~min}$. For immunoblotting, Jurkat T cells $\left(5 \times 10^{5}\right)$ were pretreated with or without O-(2-Acetamido-2-deoxy-Dglucopyranosylidenamino) N-phenylcarbamate (PUGNAc) (Toronto Research Chemicals, North York, Canada) for $4 \mathrm{hr}$ and stimulated for the indicated time periods in the presence or absence of $N$. fowleri. After incubation, cells were lysed using lysis buffer (50 mM Tris-HCl, pH 8.0; $150 \mathrm{mM} \mathrm{NaCl}, 1 \%$ NP-40, $0.5 \%$ sodium deoxycholate, $0.1 \%$ SDS, 1 mM ethylenediaminetetraacetic acid, $1 \mathrm{mM}$ phenylmethylsulfonyl fluoride, $1 \mathrm{mM}$ sodium orthovanadate, and protease inhibitor cocktail) on ice for $30 \mathrm{~min}$. Cell lysates were centrifuged at 15,000 g for $5 \mathrm{~min}$, loaded onto an SDS-PAGE gel, and transferred onto a polyvinylidene fluoride membrane (Millipore, Billerica, Massachusetts, USA), followed by blocking with 5\% skim milk. The blot was probed with primary antibodies at $4^{\circ} \mathrm{C}$ overnight, followed by incubation with appropriate secondary horseradish peroxidase-conjugated antibodies. Immunoreactivity was detected using LumiGLO (Cell Signaling Technology, Danvers, Massachusetts, USA). Mouse anti-OGlcNAc and rabbit anti- $\beta$-actin monoclonal antibodies were purchased from Abcam (Cambridge, UK) and Cell Signaling Technology (Beverly, Massachusetts, USA), respectively.
A

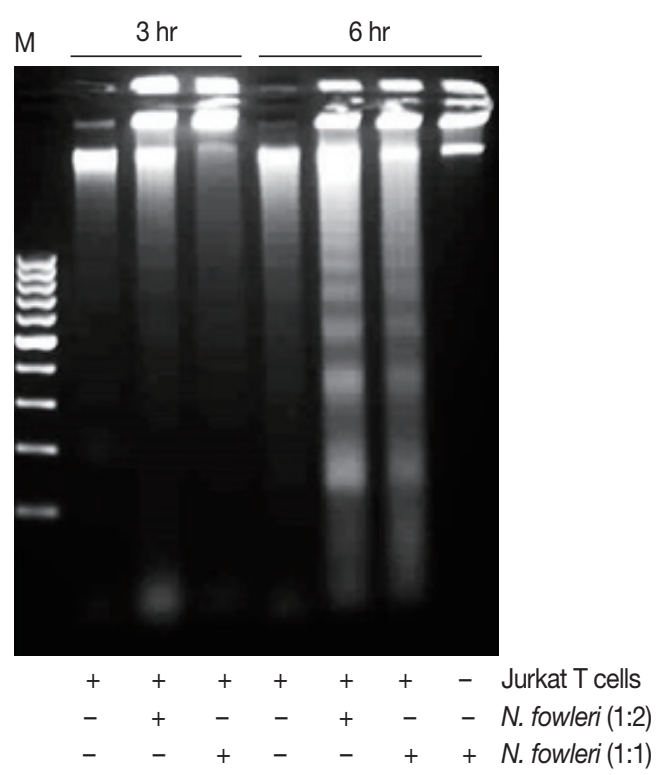

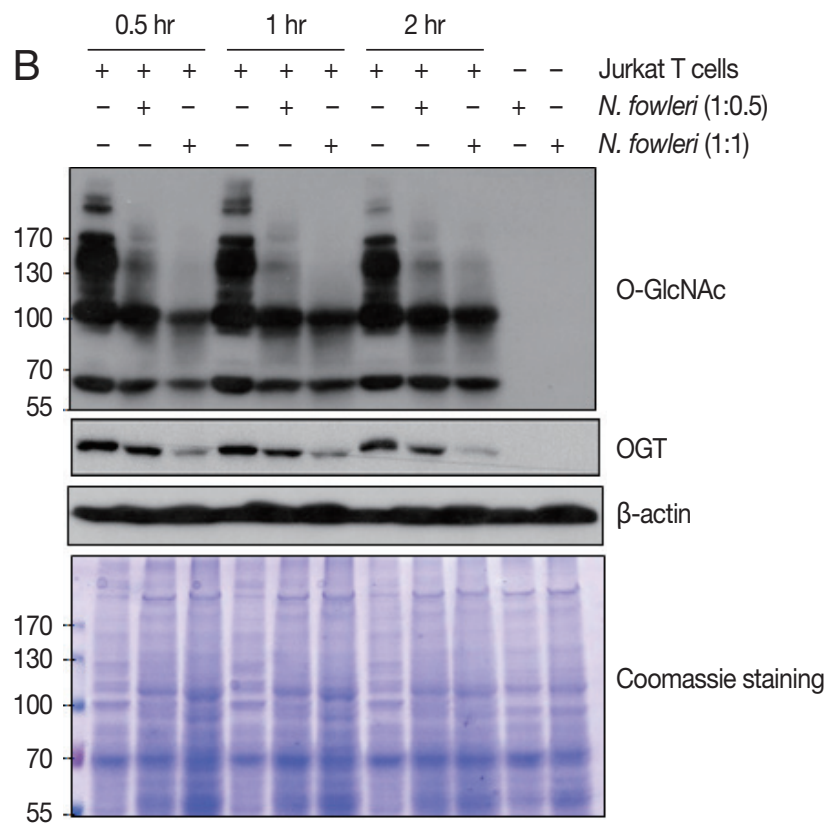

Fig. 1. Naegleria fowleri induced DNA fragmentation and O-deGlcNAcylation in Jurkat T cells. (A) Jurkat T cells (4×10\%/sample) were incubated for 3 or $6 \mathrm{hr}$ at $37^{\circ} \mathrm{C}$ with or without $\mathrm{N}$. fowleri at a ratio of 1:1 or 1:2 (Jurkat T cells to N. fowleri). DNA fragmentation was visualized with $2 \%$ agarose gel electrophoresis. An equal number of $N$. fowleri and host cells were incubated in medium alone as the negative control. M:100 bp DNA marker. (B) Jurkat T cells $\left(1 \times 10^{6} /\right.$ sample) were incubated for various time periods at $37^{\circ} \mathrm{C}$ with or without $\mathrm{N}$. fowleri at a ratio of 1:0.5 or 1:1 (Jurkat T cells to $\mathrm{N}$. fowleri). After incubation, whole cell lysates were subjected to SDS-PAGE and blotted with antibodies against $\mathrm{O}-$ GlcNAc and $\beta$-actin. The figure is representative of 3 experiments showing similar results. 
Co-incubation with $N$. fowleri trophozoites induced cell death and O-deGlcNAcylation in Jurkat T cells. As shown in Fig. 1A, N. fowleri-induced DNA fragmentation was observed in cells incubated at a ratio of 1:2 or 1:1 (Jurkat T cells to $N$. fowleri) for 3 or $6 \mathrm{hr}$. DNA fragmentation occurred in a timeand dose-dependent manner; co-incubation of Jurkat T cells with amoebae for $6 \mathrm{hr}$ at a ratio of 1:2 induced robust DNA fragmentation.
Next, we investigated whether co-incubation with $N$. fowleri induced the modulation of O-GlcNAcylated proteins. SDSPAGE results (Fig. 1B) showed that patterns of protein bands of various sizes were almost equivalent between cells incubated with or without $N$. fowleri. However, the results of immunoblotting with anti-O-GlcNAc antibody indicated a marked decrease in the level of O-GlcNAcylated proteins in Jurkat T cells incubated for $0.5 \mathrm{hr}$ with $N$. fowleri at a ratio of 1:0.5 or

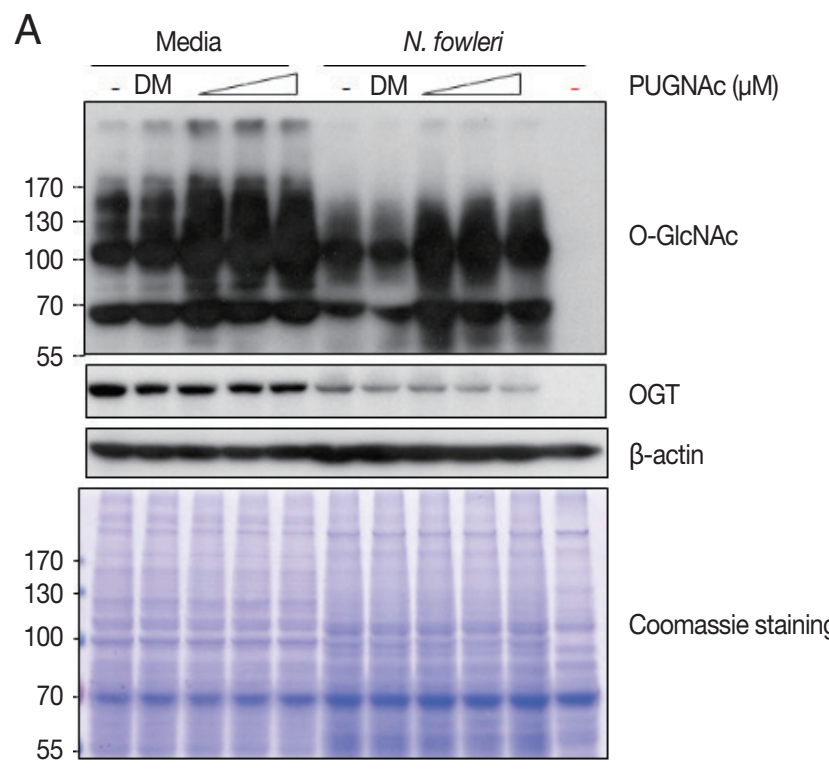

B
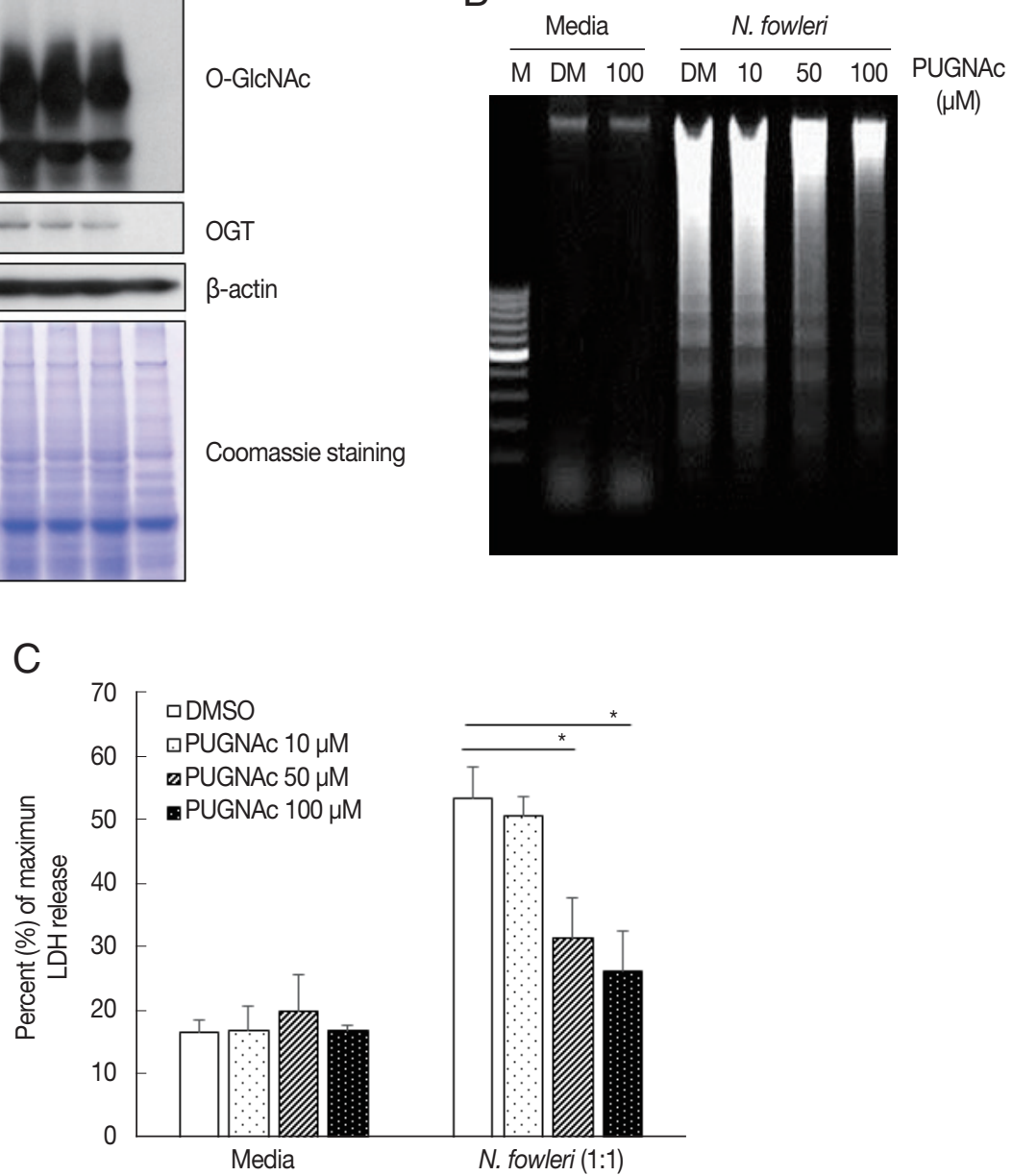

Fig. 2. Effect of OGA inhibitor on $N$. fowleri-induced $O$-deGlcNAcylation and DNA fragmentation in Jurkat $T$ cells. (A) Jurkat $T$ cells $\left(1 \times 10^{6}\right.$ sample) pretreated with PUGNAc $(10,50$, or $100 \mu \mathrm{M})$ for $4 \mathrm{hr}$ were incubated with or without $\mathrm{N}$. fowleri at a 1:1 ratio $(\mathrm{Jurkat} \mathrm{T}$ cells to $\mathrm{N}$. fowleri) for $30 \mathrm{~min}$ at $37^{\circ} \mathrm{C}$ in a $\mathrm{CO}_{2}$ incubator. DMSO $(0.5 \% \mathrm{v} / \mathrm{v})$ was used as a vehicle control. After incubation, whole cell lysates were subjected to SDS-PAGE and blotted with anti-O-GIcNAc and $\beta$-actin Ab. (B) Jurkat T cells $\left(4 \times 10^{6} /\right.$ sample) were incubated for $3 \mathrm{hr}$ at $37^{\circ} \mathrm{C}$ in the presence or absence of $N$. fowleri at a ratio of $1: 1$ (Jurkat T cells to $N$. fowleri). DNA fragmentation was visualized with $2 \%$ agarose gel electrophoresis. An equal number of $N$. fowleri and host cells were incubated in medium alone as the negative control. M: 100 bp DNA marker. DM: 0.5\% (v/v) DMSO as a vehicle control. (C) Effect of PUGNAc on N. fowleri-induced LDH release. Jurkat T cells $\left(2 \times 10^{5}\right.$ cells/sample) pretreated with PUGNAc or $0.25 \%$ DMSO (v/v) were incubated for 3hr with $N$. fowleri at a ratio of $1: 1$ (Jurkat T cells to $N$. fowleri). After incubation, supernatant was collected, and LDH released in supernatant was assayed using a CytoTox96 TM assay kit. Relative cytotoxicity (\%) was calculated along with released LDH. ${ }^{*} P<0.05$ was considered significant. 
1:1 (Jurkat T cells to $N$. fowleri), compared to the level in cells incubated with medium alone. In particular, high-MW ( $>100$ $\mathrm{kDa}$ ) O-GlcNAcylated proteins disappeared from cells in the presence of $\mathrm{N}$. fowleri, in a manner that was dependent on the amoeba load. In contrast, when N. fowleri alone was incubated with anti-O-GlcNAc antibody, in the absence of Jurkat T cells, no reacted protein bands were observed.

To examine whether OGA is involved in N. fowleri-induced O-deGlcNAcylation, we used the OGA inhibitor PUGNAc to prevent the activity of OGA in Jurkat T cells. As shown in Fig. $2 \mathrm{~A}$, the level of O-GlcNAcylated protein increased in restingstate Jurkat T cells pretreated with PUGNAc for $4 \mathrm{hr}$, compared to that in the vehicle control. In addition, pretreatment with PUGNAc prevented N. fowleri-induced O-deGlcNAcylation.

We next investigated whether OGA inhibition using PUGNAc affects Jurkat $T$ cell death induced by $N$. fowleri. As shown in Fig. 2B, pretreatment with $50 \mu \mathrm{M}$ or $100 \mu \mathrm{M}$ PUGNAc inhibited $N$. fowleri-induced DNA fragmentation compared to the control. Similarly, pretreatment of host cells with PUGNAc also inhibited $N$. fowleri-induced LDH release $(50 \mu \mathrm{M}$ : $31.5 \pm$ $6.2 \% ; 100 \mu \mathrm{M}: 26.1 \pm 6.5 \%)$ compared to results for cells pretreated with DMSO (53.5 $\pm 4.7 \%)$ (Fig. $2 \mathrm{C}$ ). These results suggest that O-deGlcNAcylation is closely related to the N. fowleri-induced Jurkat $\mathrm{T}$ cell death process.

Homeostasis between O-GlcNAcylation and O-deGlcNAcylation in mammalian cells is maintained through the balance of OGT and OGA activity [5,6]. For example, an increase in the level of O-GlcNAcylated protein is associated with increased OGT activity or decreased OGA activity. Conversely, a decrease in the level of O-GlcNAcylated protein is associated with decreased OGT expression or increased OGA expression $[6,8,10]$. Here, we showed that $N$. fowleri caused a decrease in the O-GlcNAcylated protein level in Jurkat T cells in a dose-dependent manner within $30 \mathrm{~min}$. The OGT expression in Jurkat $\mathrm{T}$ cells was gradually reduced upon co-incubation with $\mathrm{N}$. fowleri (Fig.1A). This result is consistent with the recent report that protozoan parasite E. histolytica induces O-deGlcNAcylation by decreasing OGT expression during host cell death. However, the expression level of OGA of host cells was relatively not changed in Entamoeba-treated HepG2 cells [9]. Therefore, we investigated the signaling mechanism responsible for N. fowleri-induced O-deGlcNAcylation using the OGA inhibitor PUGNAc. Pretreatment of Jurkat cells with PUGNAc prevented the decrease in the level of O-GlcNAcylated proteins, which was consistent with a recent report of an E. histo- lytica-induced host cell death model [9]. These results suggest that imbalance between OGT and OGA activity is highly involved in N. fowleri-induced O-deGlcNAcylation in Jurkat $\mathrm{T}$ cells.

The alteration of intracellular O-GlcNAcylated protein levels by cellular stimuli/stress is related to cell fate because OGlcNAcylated proteins are tightly regulated to maintain homeostasis in the resting state [11]. Increased levels of OGlcNAcylated proteins are associated with cell proliferation, survival, and activation, whereas decreased protein levels are associated with cell death, damage, and deactivation [6-8]. Several studies have reported that an increase in O-GlcNAcylated protein levels protects against cell death and various cellular stresses. For example, the addition of glucosamine leads to increased OGT expression, thereby increasing cellular resistance to stimulation and decreasing cell death rates $[6,7]$. Additionally, inhibition of OGA activity using a pharmacological OGA inhibitor, such as streptozotocin or PUGNAc, attenuates the cell death process $[12,13]$. According to a recent report, human $\mathrm{T}$ lymphoblastic cells treated with PUGNAc showed significant reductions in tributyltin-induced apoptosis [14]. In addition, Entamoeba-induced cell death was significantly prevented by pretreatment with an OGA inhibitor [9].

In conclusion, we demonstrated for the first time that $\mathrm{N}$. fowleri-induced O-deGlcNAcylation in Jurkat T cells contributes to host cell death. Our study demonstrates a novel analysis of the mechanism underlying $N$. fowleri-induced host cell death, which may be helpful in elucidating the pathogenesis of $N$. fowleri infections in humans.

\section{ACKNOWLEDGMENT}

This research was supported by a faculty research grant from Yonsei University College of Medicine (6-2015-0063).

\section{CONFLICT OF INTEREST}

The authors declare no conflict of interest related to this study.

\section{REFERENCES}

1. Jahangeer M, Mahmood Z, Munir N, Waraich UE, Tahir IM, Akram M, Ali Shah SM, Zulfqar A, Zainab R. Naegleria fowleri: Sources of infection, pathophysiology, diagnosis, and manage- 
ment; a review. Clin Exp Pharmacol Physiol 2020; 47: 199-212. https://doi.org/10.1111/1440-1681.13192

2. Kim JH, Kim D, Shin HJ. Contact-independent cell death of human microglial cells due to pathogenic Naegleria fowleri trophozoites. Korean J Parasitol 2008; 46: 217-221. https://doi.org/ 10.3347/kjp.2008.46.4.217

3. Kang SY, Song KJ, Jeong SR, Kim JH, Park S, Kim K, Kwon MS, Shin HJ. Role of the Nfa1 protein in pathogenic Naegleria fowleri cocultured with CHO target cells. Clin Diagn Lab Immunol 2005; 12: 873-876. https://doi.org/10.1128/CDLI.12.7.873-876. 2005

4. Song KJ, Jang YS, Lee YA, Kim KA, Lee SK, Shin MH. Reactive oxygen species-dependent necroptosis in Jurkat $\mathrm{T}$ cells induced by pathogenic free-living Naegleria fowleri. Parasite Immunol 2011; 33: 390-400. https://doi.org/10.1111/j.1365-3024.2011.01297.x

5. Vosseller K, Sakabe K, Wells L, Hart GW. Diverse regulation of protein function by O-GlcNAc: a nuclear and cytoplasmic carbohydrate post-translational modification. Curr Opin Chem Biol 2002; 6: 851-857. https://doi.org/ 10.1016/s1367-5931(02)003848

6. Hart GW, Housley MP, Slawson C. Cycling of O-linked beta-Nacetylglucosamine on nucleocytoplasmic proteins. Nature 2007: 446: 1017-1022. https://doi.org/10.1038/nature05815

7. Zou L, Yang S, Champattanachai V, Hu S, Chaudry IH, Marchase $\mathrm{RB}$, Chatham JC. Glucosamine improves cardiac function following trauma-hemorrhage by increased protein O-GlcNAcylation and attenuation of NF- $\{$ kappa $\}$ B signaling. Am J Physiol Heart Circ Physiol 2009; 296: 515-523. https://doi.org/10.1152/ ajpheart.01025.2008

8. Love DC, Hanover JA. The hexosamine signaling pathway: deciphering the "O-GlcNAc code". Sci STKE 2005; 2005: re13. https:// doi.org/10.1126/stke.3122005re13

9. Lee YA, Min A, Shin MH. O-deGlcNAcylation is required for Entamoeba histolytica-induced HepG2 cell death. Microb Pathog 2018; 123: 285-295. https://doi.org/10.1016/j.micpath.2018.07.012

10. Butkinaree C, Park K, Hart GW. O-linked beta-N-acetylglucosamine (O-GlcNAc): Extensive crosstalk with phosphorylation to regulate signaling and transcription in response to nutrients and stress. Biochim Biophys Acta 2010; 1800: 96-106. https://doi. org/10.1016/j.bbagen.2009.07.018

11. de Queiroz RM, Madan R, Chien J, Dias WB, Slawson C. Changes in O-Linked N-Acetylglucosamine (O-GlcNAc) Homeostasis Activate the p53 Pathway in Ovarian Cancer Cells. J Biol Chem 2016; 291: 18897-18914. https://doi.org/10.1074/jbc.M116.734533

12. Pathak S, Dorfmueller HC, Borodkin VS, van Aalten DM. Chemical dissection of the link between streptozotocin, $\mathrm{O}$ GlcNAc, and pancreatic cell death. Chem Biol 2008; 15: 799807. https://doi.org/10.1016/j.chembiol.2008.06.010

13. Ngoh GA, Watson LJ, Facundo HT, Jones SP. Augmented OGlcNAc signaling attenuates oxidative stress and calcium overload in cardiomyocytes. Amino Acids 2011; 40: 895-911. https:// doi.org/10.1007/s00726-010-0728-7

14. Johnson B, Opimba M, Bernier J. Implications of the O-GlcNAC modification in the regulation of nuclear apoptosis in T cells. Biochim Biophys Acta 2014; 1840: 191-198. https://doi.org/10.1016/ j.bbagen.2013.09.011 
